\title{
Er:YAG Laser: Antimicrobial Effects in the Root Canals of Dogs' Teeth with Pulp Necrosis and Chronic Periapical Lesions
}

\author{
MÁRIO R. LEONARDO, D.D.S., Ph.D., ${ }^{1}$ M.G. GUILLÉN-CARÍAS, D.D.S., M.S., ${ }^{2}$ \\ J.D. PÉCORA, D.D.S., Ph.D., ${ }^{3}$ I.Y. ITO, Ph.D., ${ }^{4}$ and L.A.B. SILVA, D.D.S., Ph.D. ${ }^{1}$
}

\begin{abstract}
Objective: Our goal in this study was to evaluate the antimicrobial effect of Er:YAG laser applied after biomechanical preparation of the root canals of dog's teeth with apical periodontitis. Background Data: Various in vitro studies have reported effective bacterial reduction in infected root canals using Er:YAG laser. However, there is no in vivo research to support these results. Methods: Forty root canals of dogs' premolar teeth with pulp necrosis and chronic periapical lesions were used. An initial microbiological sample was taken, and after biomechanical preparation was carried out, a second microbiological sample was taken. The teeth were divided into two groups: Group I-biomechanical preparation was taken of root canals without Er:YAG laser application; Group II-biomechanical preparation was taken of root canals with Er:YAG laser application using 140-mJ input, 63-mJ output/15 Hz. After coronal sealing, the root canals were left empty for 7 days at which time a third microbiological sample was taken. The collected material was removed from the root canal with a \#40 K file and placed in transport media. It was serially diluted and seeded on culture dishes selective for anaerobes, aerobes, and total streptococci. Colony-forming units per milliliter $(\mathrm{CFU} / \mathrm{mL})$ were counted. Results: Groups I and II showed an increase of CFU/mL for all microorganisms 7 days after treatment, being statistically significant for anaerobes in Group I and for anaerobes and total streptococci in Group II. When comparing CFU/mL of Groups I and II, there was a statistically significant increase after $7 \mathrm{~d}$ for total streptococci in Group II. Conclusion: Er:YAG laser applied after biomechanical preparation did not reduce microorganisms in the root canal system.
\end{abstract}

\section{INTRODUCTION}

D ENTAL PULP NECROSIS facilitates the access of microorganisms to the root canal, producing favorable conditions for bacterial multiplication and proliferation. In these cases, obligate anaerobes constitute a restricted group of microorganisms that prevail in this environment. ${ }^{1-4}$ With the persistence of infection, microorganisms and their enzymes and toxins propagate through the entire root canal system-dentinal tubules, secondary, accessory, and lateral canals, collateral ramifications, and apical deltas. 5
The periapical region, close to the root canal, is also reached by the persistent microorganisms and their toxins, enzymes, and endotoxins and by the products of pulp tissue disintegration. This causes a periapical lesion, which is radiographically visible. 5 Teeth with pulp necrosis and periapical lesions have a less favorable prognosis than those without periapical lesions. ${ }^{5}$ This lack of success is attributed to the predominantly anaerobic microbiota that persists in the dentinal tubules, particularly in the accessory canals of the apical third, and also to the presence of extraradicular infection and apical bacterial biofilm. ${ }^{2,6-8}$

\footnotetext{
1Department of Endodontics, School of Dentistry of Araraquara, UNESP, Araraquara, SP, Brazil.

${ }^{2}$ Department of Pediatric, Preventive, and Social Dentistry, School of Dentistry of Ribeirão Preto, University of São Paulo, Ribeirão Preto, SP, Brazil.

${ }^{3}$ Department of Restorative Dentistry, School of Dentistry of Ribeirão Preto, University of São Paulo, Ribeirão Preto, SP, Brazil.

${ }^{4}$ Department of Clinical, Toxicological, and Bromatological Analysis, School of Pharmaceutical Sciences of Ribeirão Preto, University of São Paulo, Ribeirão Preto, SP, Brazil.
} 
These facts justify the use of intracanal medication applied topically between sessions as an adjuvant of biomechanical preparation. Calcium hydroxide-based pastes have been used with the objective of eliminating persistent microorganisms in the root canal system after biomechanical preparation.5,9 More recently, researchers have focused on the antimicrobial effects of Er:YAG laser, which has been used with conventional endodontic therapy. ${ }^{10-15}$ However, studies carried out with this type of laser only report experiences in vitro. Thus, the present study evaluated the antimicrobial effect of Er:YAG laser applied in vivo after biomechanical preparation in root canals of dogs' teeth with pulp necrosis and chronic periapical lesions.

\section{MATERIALS AND METHODS}

This study was conducted in two adult 1-year-old mongrel dogs of both sexes (wt: $10-15 \mathrm{~kg}$ ), after approval by the Ethics Commission in the Use of Animals (03.1.489.53.5) of the University of São Paulo. The procedures were carried out on the second and third maxillary premolars and on the second, third, and fourth mandibular premolars for a total of 40 roots.

The animals were anesthetized intravenously with a thiopental solution (Thionembutal, $30 \mathrm{mg} / \mathrm{kg}$ body wt; Abbott Laboratories, São Paulo, Brazil). During surgery, the animals were maintained with a $0.9 \%$ chloride solution (Glicolabor Ind. Farm. Ltda., São Paulo, Brazil) administered intravenously. After standard radiographic assessment, access was made. The working length was determined as $2 \mathrm{~mm}$ short of the radiographic apex using \#30 K files. The root pulp was removed and the root canals were left exposed to the oral environment for 7 days to allow microbial contamination.

After this period, debris was removed under general anesthesia, and the root canals were irrigated with saline and dried. A cotton pledget was placed in the occlusal openings, which were sealed with zinc oxide-eugenol cement (IRM; Dentsply, Rio de Janeiro, Brazil). Radiographs were taken at 15-day intervals to monitor the development of apical periodontitis, which was observed after 45-60 days.

After isolation of a hemiarch with a rubber dam, the teeth and operative field were disinfected with a $1.0 \%$ chlorhexidine digluconate solution under general anesthesia. After removal of the temporary sealing and cotton pledget, the first sample for microbiological examination was taken with a \#40 $\mathrm{K}$ file (Maillefer, Ballaigues, Switzerland) placed in the root canals at the working length. Subsequently, the root canals were submitted to progressive neutralization of toxic/septic content throughout, using $\mathrm{K}$ files and abundant irrigation, aspiration, and inundation with a $1 \%$ sodium hypochlorite solution (Laboratório de Química Analítica do Departamento de Física e Química, Faculdade de Ciências Farmacêuticas de Ribeirão Preto, Ribeirão Preto, Brazil). The apical foramen was enlarged by sequential use of \#15 to \#30 K files to the radiographic apex, irrigating with $1 \%$ sodium hypochlorite between files. Instrumentation was then performed to the working length up to a \#70 K file for the root canal of mandibular teeth and a $\# 60 \mathrm{~K}$ file for the root canal of maxillary teeth. After each instrument change, abundant irrigation, aspiration, and inundation of the root canal was performed using a $1 \%$ sodium hypochlorite solution. A \#30 K file was used the total length of the root to ensure that no dentin chips or other residue remained in the apical foramen. The root canals were dried with sterile absorbent paper points (Tanari Industrial Ltda., Manaus, Brazil) and filled with a $14.3 \%$ ethylenediaminetetraacetic acid solution, buffered at pH 7.4 (EDTA, Odahcan, Herpo Produtos Dentários Ltda., Rio de Janeiro, Brazil) and shaken for 3 min. After abundant irrigation with $1 \%$ sodium hypochlorite solution of EDTA, the root canals were dried and the second sample for microbiological examination was taken with a \#40 K file.

The root canals were divided into two groups: Group I (experimental)—20 root canals were subjected only to biomechanical preparation; Group II (experimental) - 20 root canals were irradiated after biomechanical preparation using an Er:YAG laser (Kavo Key Laser 1242, Kavo Dental GmbH, Vertriebsgesellschaft, Germany) at the Laboratory of Endodontic Research of the Ribeirão Preto Dental School, University of São Paulo. An E2055 handpiece and 50/28 optical fiber with a diameter of $0.285 \mathrm{~mm}$ and transmission factor of 0.45 were used. The optical fiber was inserted as far as the working length, the laser was activated, and the root canals were irradiated with circular movements, from apical to cervical at $\sim 2 \mathrm{~mm} / \mathrm{s}$. Four irradiations of $10 \mathrm{~s}$ each were applied, with intervals of $30 \mathrm{~s}$ between each. During the intervals, the root canals were filled with deionized distilled water. The settings used were $15 \mathrm{~Hz}, 140-\mathrm{mJ}$ input (energy shown on the display), 63 output (energy irradiated by the optical fiber). The international security standards were observed: use of appropriate lenses, gloves, mask, room with locked door, and warning light outside the room. After irradiation, the root canals were left empty.

After treatment, occlusal openings in both groups were sealed with zinc oxide-eugenol cement for $7 \mathrm{~d}$. After this period, isolation and disinfection of the operative field using $1.0 \%$ chlorhexidine digluconate solution were performed under general anesthesia. The temporary seal was removed and the third sample for microbiological examination was taken with a $\# 40 \mathrm{~K}$ file. For the three bacteriologic samples, the active parts of the files were separated from their handles and placed in test tubes containing 2-mL RTF for microbiological processing.

\section{Microbiological procedures}

The microbiological procedures were carried out at the Laboratory of Clinical, Toxicological, and Bromatological Analysis of the School of Pharmaceutical Sciences of Ribeirão Preto, University of São Paulo. Under aseptic conditions, sterilized glass pearls were added to the test tubes containing the files' working parts. The test tubes were vibrated (Mixtron-Toptronix) for $3 \mathrm{~min}$ at maximum speed and afterward submitted to a serial decimal dilution down to $10^{-5}$ in PBS. Fifty $\mu \mathrm{L}$ of these dilutions were seeded in culture media: Ask (tryptic soy agar, Difco, Detroit, MI), As (brain heart infusion, Difco), Ms (Mitis salivarius agar, Difco). The Ask plates were incubated anaerobically using the Anaerobac system for $7-10$ days at $37^{\circ} \mathrm{C}$. The As plates were incubated aerobically for $24-48 \mathrm{~h}$ at $37^{\circ} \mathrm{C}$, and the Ms plates were incubated in capnophilia using the expired air system for $48-72 \mathrm{~h}$ at $37^{\circ} \mathrm{C}$. After the incubation period, the plates were evaluated with a stereoscopic microscope (Nikon, 
Japan) under reflected light and the number of colony-forming units per milliliter $(\mathrm{CFU} / \mathrm{mL})$ were counted and recorded. Moreover, $5 \mathrm{~mL}$ thioglycolate medium (without dextrose or indicator, Difco) was added to the test tubes containing the remaining suspension and the files' working parts. These were incubated until turbidity, or up to 20 days. This procedure allows the detection of microorganisms present in quantities $<20 \mathrm{CFU} / \mathrm{mL}$.

\section{Statistical analysis}

Statistical analysis was performed with the Graphpad Prism program (San Diego, CA). The Mann-Whitney and Wilcoxon tests were used to compare $\mathrm{CFU} / \mathrm{mL}$ counts of anaerobes, aerobes, and total streptococci in Groups I and II before biomechanical preparation and 7 days after treatment.

\section{RESULTS}

At baseline, Groups I and II showed an elevated number for all microorganisms. When compared before treatment, the medians of CFU/mL in Group II were greater than those of Group I, being statistically significant for aerobes $(p=0.0338)$. Thioglycolate medium showed $100 \%$ positive cultures in both groups before and after treatment. Immediately after biomechanical preparation, there was a complete elimination of microorganisms in both Groups I and II. However, thioglycolate medium detected microorganisms in numbers $<20 \mathrm{CFU} / \mathrm{mL}$ in $22.2 \%$ cases for Group I and $23 \%$ for Group II. Seven days after treatment, the number of microorganisms was greater in Group II than in Group I, being statistically significant for total streptococci $(p=0.0275)$. Seven days after treatment, the number of microorganisms in Group I increased, being statistically significant for anaerobes (Table $1 ; p=0.0258$ ). Group II had an increase 7 days after treatment that was statistically significant for anaerobes and total streptococci (Table 1).

\section{DISCUSSION}

Endodontic infections are polymicrobial and over time present qualitative and quantitative changes. These alterations lead to the establishment of predominantly obligate anaerobic microbiota. In the present study, a large quantity of microorganisms was present. In Group I, the predominant microorganisms were anaerobes, present in $100 \%$ of the cases, followed by aerobes $(94.4 \%)$ and total streptococci $(88.9 \%)$. Group II showed an initial prevalence of $100 \%$ positive cultures for all microorganisms before biomechanical preparation, being statistically significant for aerobes compared to Group I. Thioglycolate medium was positive in $100 \%$ of the cases in both Groups I and II.

The intention of biomechanical preparation by irrigation with antibacterial solutions is to reduce the number of bacteria in the root canal. ${ }^{9}$ In this study, immediately after biomechanical preparation, instrumentation followed by abundant irrigation was effective in reducing the number of microorganisms compared to their initial prevalence.

According to the results comparing the initial prevalence and 7 days after treatment, there was an increase in microorganisms. These results do not agree with various studies in the literature that report high success rates for the bactericidal effect of Er:YAG laser when used in root canals. ${ }^{10,12,13,15}$ The difference between these results is the previous lack of an adequate in vitro reproduction of root canal and periapical region conditions. These conditions are favorable for the growth and proliferation of the remaining bacteria in the root canal system. In an endodontic infection, microorganisms are not limited to only the main root canal lumen. The lateral, secondary, and accessory canals, as well as the dentinal tubules, are progressively invaded by bacteria ${ }^{5}$ and by apical bacterial biofilm. ${ }^{16}$

In the present work, the root canals were left empty with a temporary coronal seal for 7 days after biomechanical preparation and laser application to permit proliferation of the remain-

Table 1. Comparison of CFU/mL Counts of Anaerobes, Aerobes, and Total Streptococci in Group I (BIOMEChanical Preparation OnLY) AND GRoup II (LASER GRouP) AT BASELINE AND FOLLOW-UP

\begin{tabular}{cccc}
\hline & Anaerobes & Aerobes & Total streptococci \\
\hline Group I & & & \\
Baseline & 400 & 185 & 60 \\
& $(40-10000)$ & $(0-4000)$ & $(0-4500)$ \\
Follow-up & 1425 & 315 & 145 \\
& $(0-366000)$ & $(0-410000)$ & $(0-90000)$ \\
$p$ value & 0.0258 & 0.4791 & 0.3623 \\
Group II & & & 170 \\
Baseline & 1,120 & 680 & $(40-2520)$ \\
& $(20-16600)$ & $(40-7660)$ & 3,600 \\
Follow-up & 24,000 & 13,120 & $(0-122000)$ \\
$p$ value & $(0-118000)$ & $(0-126000)$ & 0.0134 \\
\hline
\end{tabular}

Data are reported as median (range). $p$ value for the difference between baseline and follow-up of each group: Wilcoxon test. 
ing microorganisms in the root canal system, which can reach numbers equivalent to baseline. This differs from the study by Mehl et al.; ${ }^{12}$ they waited $2 \mathrm{~h}$ between bacterial inoculation and laser application, without subjecting the inoculated dentinal samples to incubation. Stephan et al. ${ }^{15}$ and Moritz et al. ${ }^{13}$ subjected the samples inoculated with bacteria to incubation for only 24 and $48 \mathrm{~h}$, respectively, time considered insufficient for bacterial proliferation.

A factor that could have caused the absence of an antimicrobial effect in the present study is that Er:YAG laser radiation is absorbed by hydroxyapatite, thereby not reaching the remaining bacteria in the dentinal tubules and periapical lesion. Jelínková et al. ${ }^{11}$ evaluated the propagation of laser light in the root canal with the aid of an energy detector placed on the external root surface of human teeth. No energy was detected, meaning that Er:YAG laser radiation was completely absorbed by the root canal walls and did not reach the apex ramifications. Laser radiation did not penetrate the surrounding tissues of the root canal. Matsumoto ${ }^{17}$ believes that it is impossible to "sterilize" root canals completely, without "sterilizing" the surrounding periapical tissue; in the future, sterilization by means of laser could be conducted using other medicaments.

Another factor that could have influenced the difference in our results is the period of microorganism sampling, which in our study was performed before and immediately after biomechanical preparation and 7 days after treatment. Mehl et al., ${ }^{12}$ Moritz et al., ${ }^{13}$ and Stephan et al. ${ }^{15}$ took a sample immediately after laser application, which does not demonstrate the real bacteriologic conditions of the root canal system.

One limitation of in vitro studies is the lack of nutrients for microorganisms, due to the sterilization of teeth samples, which removes fluids and thermolabile proteins essential to the remaining microorganisms in the root canal system. According to Sundqvist, ${ }^{3}$ the presence of serum-like substances is one of the most important ecologic factors in an endodontic infection. In in vivo studies, the supplement of fluids and thermolabile proteins is continuous and promotes bacterial multiplication.

In our study, the results showed an increase in the number of microorganisms after 7 days of treatment when compared to baseline in both Groups I and II. This increase was greater in Group II, than in Group I, and was statistically significant for total streptococci. This increase may be caused by many factors. In a chronic infection, there is an accumulation of microbial metabolic subproducts, such as acids and alkalis, and production of antimicrobial substances. There is also an accumulation of substances from tissue necrosis and microorganism lysis, (i.e., release of bacterial cytoplasmic content with accumulation of the involucre components, such as the cell wall of Gram-negative and Gram-positive microorganisms and the cellular membrane). Biomechanical preparation removes these substances, allowing the remaining microorganisms to come into contact with nutrients. Thus, microbial population will multiply at an exponential rate.

The removal of smear layer promotes dentinal permeability, increasing the afflux of remaining microorganisms from the dentinal tubules to the main root canal lumen. This may have influenced the results in Group II. Takeda et al. ${ }^{18}$ evaluated the effect of Er:YAG laser on root canal walls of human teeth using scanning electronic microscopy and reported the absence of debris and smear layer and open dentinal tubules.
Based on our results and after statistical analysis, we conclude that biomechanical preparation with or without Er:YAG laser did not remove the remaining microbiota from the root canal system. These results reinforce the need to conduct new studies using laser radiation with the application of intracanal dressings between sessions to obtain adequate disinfection of the root canal system in teeth with apical periodontitis.

\section{CONCLUSION}

We conclude that the antimicrobial effect in Group II (with Er:YAG laser application) was similar to that of Group I (without laser application) in the root canals of dogs' teeth with apical periodontitis.

\section{REFERENCES}

1. Akpata, E.S., and Blechman, H. (1982). Bacterial invasion of pulpal dentin wall in vitro. J. Dent. Res. 61, 435-438.

2. Ando, N., and Hoshino, E. (1990). Predominant obligate anaerobes invading the deep layers of root canal dentine. Int. Endod. J. 23, 20-27.

3. Sundqvist, G. (1992). Associations between microbial species in dental root canal infections. Oral Microbiol. Immunol. 7, 257-262.

4. Sundqvist, G. (1992). Ecology of the root canal flora. J. Endod. 18, 427-430.

5. Leonardo, M.R., Almeida, W.A., Ito, I.Y., et al. (1994). Radiographic and microbiologic evaluation of posttreatment apical and periapical repair of root canals of dogs' teeth with experimentally induced chronic lesion. Oral Surg. Oral Med. Oral Pathol. 78, 232-238.

6. Oguntebi, B.R. (1994). Dentine tubule infection and endodontic therapy implications. Int. Endod. J. 27, 218-222.

7. Sjögren, U. (1996). Success and failure in endodontics [Doctoral dissertation]. Umea, Sweden.

8. Tronstad, L., Barnett, F., Riso, K., et al. (1987). Extraradicular endodontic infections. Endod. Dent. Traumatol. 3, 86-90.

9. Leonardo, M.R., and Silva, L.A.B. (1998). Medicação tópica entre sessões, "curativo de demora" em biopulpectomia e necropulpectomias I e II, in: Endodontia: tratamento de canais radiculares. M.R. Leonardo, and J.M. Leal (eds.). São Paulo: Ed. Médica Panamericana, pp. 491-534.

10. Gutknecht, N., Schürmann, M.G., Apel, C., et al. (2000). Bactericidal effects of Er:YAG laser radiation in root canals, in: International Congress on Lasers in Dentistry, 7 (abst). Brussels: ISLD, p. 18.

11. Jelínková, H., Dostálová, T., Dušková, J., et al. (1999). Er:YAG and alexandrite radiation propagation in root canal and its effect on bacteria. J. Clin. Med. Surg. 17, 267-272.

12. Mehl, A., Folwaczny, M., Haffner, C., et al. (1999). Bactericidal effects of $2.94 \mu \mathrm{m}$ Er:YAG-laser radiation in dental canal roots. J. Endod. 25, 490-493.

13. Moritz, A., Schoop, U., Goharkhay, K., et al. (1999). The bactericidal effect of Nd:YAG, Ho:YAG, and Er:YAG laser irradiation in the root canal: an in vitro comparison. J. Clin. Med. Surg. 17, 161-164.

14. Shoji, S., Nemoto, M., Lee, B.S., et al. (2000). Evaluation of bactericidal effect in depth on canal walls after Er:YAG laser irradiation, in: Internarional Congress on Lasers in Dentistry, 7 (abst). Brussels: ISLD, p. 45. 
15. Stephan, B., Wellings, H., and Raab, W.H.M. (1999). Microbial effects of Er:YAG laser irradiation on infected root canals. J. Dent. Res. 78, 385.

16. Leonardo, M.R., Rossi, M.A., Silva, L.A.B., et al. (2002). SEM evaluation of bacterial biofilm and microorganisms on the apical external root surface of human teeth. J. Endod. 28, 295-299.

17. Matsumoto, K. (2000). Lasers in endodontics. Dent. Clin. North Am. 44, 889-906.

18. Takeda, F.H., Harashima, T., Eto, J.N., et al. (1998). Effect of Er:YAG laser treatment on the root canal walls of human teeth: an SEM study. Endod. Dent. Traumatol. 14, 270-273.
Address reprint requests to: Mário Roberto Leonardo, D.D.S., Ph.D. Departamento de Odontologia Restauradora Faculdade de Odontologia de Araraquara Universidade Estadual Julio de Mesquita Filho - UNESP Rua Humaitá, 1680 Araraquara, $S P$ Brasil 14801-903

E-mail: dciops@forp.usp.br 